\title{
The circumstellar environment of IRAS 16293-2422
}

\section{ISO-LWS and SCUBA observations}

\author{
J. C. Correia ${ }^{1}$, M. Griffin ${ }^{2}$, and P. Saraceno ${ }^{3}$ \\ 1 Departamento de Física, Faculdade de Ciências da Univ. de Lisboa, Campo Grande, 1749 - 016 Lisboa, Portugal \\ 2 Department of Physics and Astronomy, Queens Buildings, Cardiff University, PO Box 913, 5 The Parade, \\ Cardiff CF24 3YB, UK \\ e-mail: matt.griffin@astro.cf.ac.uk \\ 3 CNR - Istituto di Fisica dello Spazio Interplanetario, via Fosso del Cavaliere, 00133 Roma, Italy \\ e-mail: saraceno@lupus.ifsi.rm.cnr.it
}

Received 19 August 1999 / Accepted 14 January 2004

\begin{abstract}
We present far-infrared (FIR) continuum observations of the deeply embedded source IRAS 16293-2422 performed with the Long Wavelength Spectrometer (LWS) on-board the Infrared Space Observatory (ISO). We also report 450 and $850 \mu \mathrm{m}$ mapping observations done with the Submillimetre Common-User Bolometer Array (SCUBA) at the James Clerk Maxwell Telescope (JCMT). We combined these observations with IRAS and other JCMT data available in the literature to construct a complete spectral energy distribution (SED) of the source. A spherically symmetric dusty envelope model was used to reproduce the SED and to characterize the circumstellar matter around the object. We call attention to the fact that when using models such as the one presented here, one needs spatial information about the object to distinguish between different possible fits to the SED. A comparison between the intensity profiles at 450 and $850 \mu \mathrm{m}$ obtained from the SCUBA observations and the profiles predicted by the model allowed us to constrain the size of the envelope and its density distribution. The SED and the $850 \mu \mathrm{m}$ intensity profile of the source are consistent with a centrally peaked power law dust density distribution of the form $\rho(r) \propto r^{-p}$ with $p=1.5-2$, with a radius $R_{\mathrm{env}}=3000-3250 \mathrm{AU}$, defining a very compact circumstellar envelope. We estimate a bolometric luminosity $L_{\mathrm{bol}}=36 L_{\odot}$, an envelope mass $M_{\mathrm{env}}=3.4 M_{\odot}$, and a submillimetre to bolometric luminosity ratio $L_{\text {submm }} / L_{\text {bol }}=1.9 \%$, confirming that the source shows a submillimetre excess characteristic of Class 0 sources.
\end{abstract}

Key words. ISM: individual objects: IRAS 16293-2422 - infrared: ISM - stars: circumstellar matter

\section{Introduction}

The spectral energy distribution of a young stellar object (YSO) is determined by the amount and distribution of dust in its circumstellar environment. As an object evolves, the dust is redistributed between the infalling envelope and the eventual circumstellar disk. This means that different classes of objects, representing different evolutionary stages, have different SEDs (Adams et al. 1987). As a consequence, SEDs have been used to study the evolutionary status of YSOs. However, the SED of an object is not necessarily defined uniquely and can be reproduced by different models due to the degeneracy of various parameters, as has been noted by several authors (Thamm et al. 1994; Butner et al. 1994; Boss \& Yorke 1995; Men'shchikov \& Henning 1997). If one wants to obtain a physically meaningful fit to the SED, one has to obtain spatial information about the dust distribution and then compare it with the spatial intensity distribution predicted by the model. The analysis of the spatial

Send offprint requests to: J. C. Correia, e-mail: jcc@fc.ul.pt information is the key to help us to put reliable contraints on the source model parameters. If we want to take advantage of spatial data, we need to have a source model and a radiative transport code to predict the expected intensity distribution of the source.

Before the launch of $\mathrm{ISO}^{1}$, most of these studies relied on IRAS data to cover the FIR part of the spectrum, limiting the spectral coverage to wavelengths below $100 \mu \mathrm{m}$. However, the coolest and most deeply embedded sources, such as Class 0 objects, emit most of their radiation and have their peak emission beyond $100 \mu \mathrm{m}$ (André et al. 1993, 2000). We have used ISO-LWS data to access this region of the spectrum in order to construct a detailed SED of the protostar IRAS 16293-2422. We have also used a spherically symmetric envelope model to reproduce the SED and characterize the circumstellar matter around the object. The model allows us to predict the source intensity profiles at any chosen wavelengths. We have

\footnotetext{
${ }^{1}$ ISO is an ESA project with instruments funded by ESA Member States (especially the PI countries: France, Germany, The Netherlands and UK) with the participation of ISAS and NASA.
} 
complemented the ISO observations with SCUBA data in order to study the intensity profiles of the object and to derive integrated fluxes at 450 and $850 \mu \mathrm{m}$. These were then combined with the $80^{\prime \prime}$ beam size ISO observations in order to plot the SED. The analysis of the intensity profiles enabled us to distinguish between different possible fits to the SED and get reliable estimatives for the model parameters.

IRAS 16293-2422 is a Class 0 source (André et al. 2000) situated in an isolated and cold molecular cloud core in $\rho$ Ophiuchi at a distance of $160 \mathrm{pc}$ (Whittet 1974). This deeply embedded object, undetected at wavelengths shorter than $25 \mu \mathrm{m}$, has been the subject of many studies. Radio and interferometric observations have shown that this IRAS source is actually a binary system whose two components are $800 \mathrm{AU}$ apart (Wootten 1989; Mundy et al. 1992; Walker et al. 1993).

A complex molecular outflow was discovered independently by Walker et al. (1985), Fukui et al. (1986) and Wootten \& Loren (1987). Later, higher spatial resolution showed a quadrupole signature (Walker et al. 1988; Mizuno et al. 1990). This was later interpreted as two separated and almost perpendicular molecular outflows (Walker et al. 1993).

Mundy et al. (1986) combined their $2.7 \mathrm{~mm}$ interferometric observation with IRAS fluxes and with the $1.3 \mathrm{~mm}$ flux from Walker et al. (1986) to construct the SED of the source. By fitting the observations with a greybody they estimated a bolometric luminosity of $\simeq 27 L_{\odot}$ and a total circumstellar mass in the range 0.9-6 $M_{\odot}$. Walker et al. (1990) mapped the object at $1.3 \mathrm{~mm}$ as part of a continuum survey of cold IRAS sources. Their fit of the SED allowed them to estimate a bolometric luminosity of $\simeq 29 L_{\odot}$ and a mass of $2 M_{\odot}$. Observations at 870 and $1300 \mu \mathrm{m}$ allowed Mezger et al. (1992) to estimate an envelope mass of $3 M_{\odot}$. Later, André \& Montmerle (1994), based on a $1.3 \mathrm{~mm}$ continuum survey for cold circumstellar dust in the $\rho$ Ophiuchi cloud, estimated an envelope mass of $2.3 M_{\odot}$. These studies relied on SEDs constructed with just a few photometric points covering the far-infrared and submillimetre ranges, making it difficult to estimate the peak emission with the resulting uncertainties for the temperature distribution and bolometric luminosity.

\section{Observations}

\subsection{ISO-LWS observations}

IRAS 16293-2422 was observed with the Long Wavelength Spectrometer (Clegg et al. 1996; Swinyard et al. 1996). The observations were done in grating mode with spectral resolution elements of $0.29 \mu \mathrm{m}$ between 43 and $90 \mu \mathrm{m}$ and $0.6 \mu \mathrm{m}$ between 90 and $197 \mu \mathrm{m}$. The on-source observations were taken at the position $(\alpha, \delta)_{1950}=\left(16^{\mathrm{h}} 29^{\mathrm{m}} 20.9^{\mathrm{s}},-24^{\circ} 22^{\prime} 13^{\prime \prime}\right)$. An off-source spectrum was obtained by averaging eleven spectra taken around the on-source (see Fig. 1 of Ceccarelli et al. 1998). The flux calibration of the LWS is referred to observations of Uranus and the absolute flux calibration is better than $30 \%$ (Trams 1998). The LWS beam is about $80^{\prime \prime}$ at all wavelengths (Swinyard et al. 1996). The reduction of the
Table 1. ISO-LWS photometry of IRAS 16293-2422 $(F W H M=$ $\left.80^{\prime \prime}\right)$.

\begin{tabular}{cc}
\hline \hline $\begin{array}{c}\text { Wavelength } \\
(\mu \mathrm{m})\end{array}$ & $\begin{array}{c}\text { Flux density } \\
(\mathrm{Jy})\end{array}$ \\
\hline 50 & 150 \\
60 & 350 \\
70 & 640 \\
80 & 940 \\
90 & 1180 \\
100 & 1320 \\
125 & 1590 \\
150 & 1590 \\
175 & 1360 \\
190 & 1100 \\
\hline
\end{tabular}

LWS data was performed using ISAP ${ }^{2}$. The spectra were corrected for any fringing (a modulation of the signal, cosinusoidal in wavelength space, caused by an optical interference in the instrument, which affects some of the observations) by using a defringing algorithm written by the LWS Consortium (Swinyard et al. 1996). No attempt was made to remove the discrepancies between the ten LWS sub-spectra in order to get a single smooth spectrum as the results of this operation can be strongly dependent on the detector used as a reference and, consequently, can introduce significant errors on the final spectrum (Correia 2000). The average off-source spectrum was then subtracted from the on-source one. Finally, the data were binned into ten data points, each one representing one detector. The photometric results are summarized in Table 1.

\subsection{SCUBA observations}

The observations were made in August 1998 using the Submillimetre Common-User Bolometer Array (SCUBA) (Holland et al. 1999) at the James Clerk Maxwell Telescope $\left(\mathrm{JCMT}^{3}\right)$ on Mauna Kea, Hawaii. SCUBA consists of two bolometer arrays, the Long-Wave (LW) array comprising 37 pixels operating in the 850 and $750 \mu \mathrm{m}$ atmospheric transmission windows and the Short-Wave (SW) array with 91 pixels optimized for operation at 450 and $350 \mu \mathrm{m}$. The pixels are placed in a hexagonal pattern. The observations were taken simultaneously at 450 and $850 \mu \mathrm{m}$ using a dichroic beam splitter. The jiggle-map observing mode, intended to map small areas, was used. The full-width at half maximum

\footnotetext{
2 ISAP (The ISO Spectral Analysis Package) was developed by a collaboration involving people from the SWS and the LWS Instrument Dedicated and Support Teams (MPE, RAL, SRON, VILSPA), IAS (Orsay) and IPAC (Caltech, Pasadena).

3 The JCMT is operated by the Joint Astronomy Centre on behalf of the UK Particle Physics and Astronomy Research Council, the Netherlands Organization for Scientific Research and the Canadian National Research Council.
} 
IRAS $16293-2422$

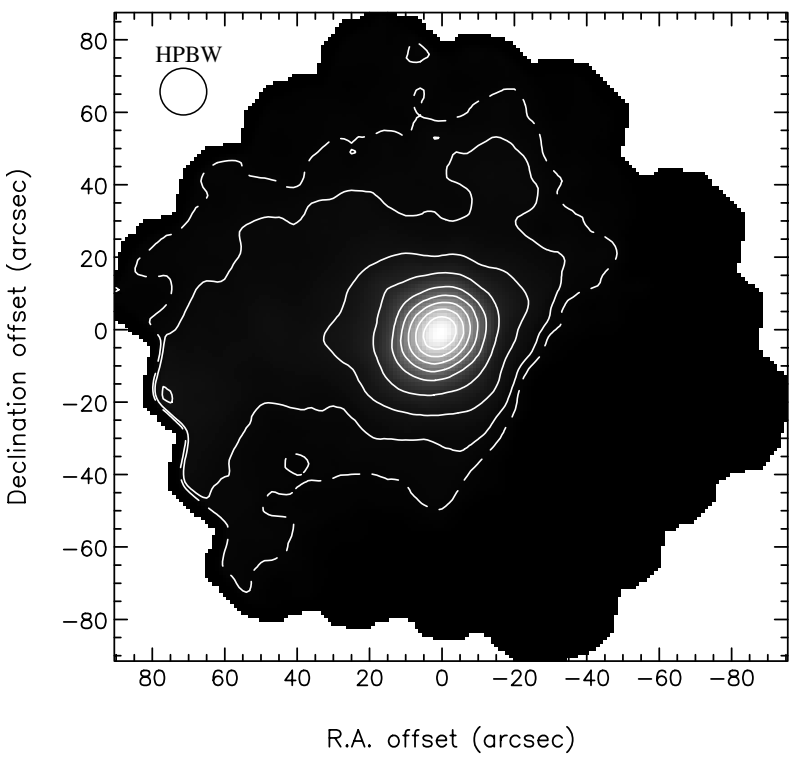

Fig. 1. $850 \mu \mathrm{m}$ image of IRAS 16293-2422. North is up and East is to the left. The hexagonal black area delimeters the area covered by SCUBA. The greyscale is linear and spans the full range of emission. The contours are at 3- $\sigma$ (dashed line), 5- $\sigma, 10-\sigma, 20-\sigma$ and then at intervals of $20-\sigma$. The $1-\sigma$ rms noise is $0.12 \mathrm{Jy} / \mathrm{beam}$. The peak emission is $16.9 \mathrm{Jy} /$ beam and the $S / N$ at the peak is $\simeq 140$. The half-power beam width of the telescope is shown by the circle.

$(F W H M)$ beam sizes were $8.8^{\prime \prime}$ and $14.6^{\prime \prime}$ at 450 and $850 \mu \mathrm{m}$, respectively.

The image at $850 \mu \mathrm{m}$ is presented in Fig. 1.

Like the LWS observations described above, the calibration is referred to observations of Uranus. The pointing of the telescope was checked before and after the observations on a nearby quasar. The observations were done using a chop throw of $120^{\prime \prime}$ in azimuth and a chop frequency of $7.8 \mathrm{~Hz}$. The atmospheric transmission was monitored by performing skydips along the night. The data were analysed using the SCUBA User Reduction Facility (SURF) (Jenness \& Lightfoot 1997). The images were initially flatfielded and corrected for extinction. The sky emission was then removed and data from bad bolometers were discarded. Finally, the maps were cleared of any spikes.

The black area indicates the region covered by the SCUBA map. The map shows a slightly elongated dust emission oriented SE-NW with a PA of $145^{\circ}$. This marginal elongation is parallel to the separation of the binary system (Wootten 1989; Mundy et al. 1992). This centrally peaked emission is surrounded by a more extended dust emission spreading towards East. This emission is associated with the separate $\mathrm{NH}_{3}$ core discussed by Wootten \& Loren (1987) which lies only $80^{\prime \prime}$ away from IRAS 16293-2422. The position of the chop of our observation is $72^{\prime \prime}$ and $96^{\prime \prime}$ away from IRAS 16293-2422 in RA and Dec, respectively, which allows us to say that our maps were not affected by this neighbouring $\mathrm{NH}_{3}$ core.

The $450 \mu \mathrm{m}$ image is shown in Fig. 2. The map is more irregular in this case due to the lower signal-to-noise.

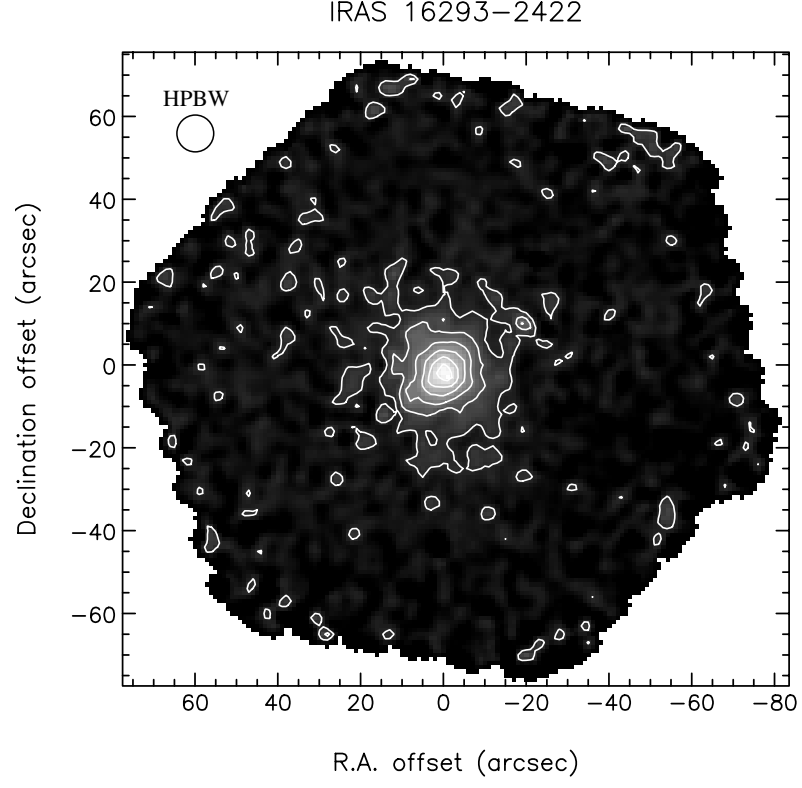

Fig. 2. $450 \mu \mathrm{m}$ image of IRAS 16293-2422. North is up and East is to the left. The hexagonal black area delimiters the area covered by SCUBA. The greyscale is linear and spans the full range of emission. The contours are at 3- $\sigma$ intervals starting from 3- $\sigma$. The $1-\sigma$ rms noise is $4.5 \mathrm{Jy} / \mathrm{beam}$. The peak emission is $89.6 \mathrm{Jy} / \mathrm{beam}$ and the $S / N$ at the peak is $\simeq 20$. The half-power beam width of the telescope is shown by the circle.

Figure 3 shows the radial intensity profiles of IRAS $16293-2422$ at $850 \mu \mathrm{m}$ and $450 \mu \mathrm{m}$ as measured from the maps shown in Figs. 1 and 2. The analysis of the intensity profiles derived from the SCUBA maps seem to suggest an apparent break at $R \simeq 20^{\prime \prime}$, indicating that the envelope merges with the background at a distance corresponding to $\simeq 3000 \mathrm{AU}$ from the centre of the emission. We will use this fact as a constraint of the radius of the envelope. Based on this analysis, integrated fluxes were obtained from the maps using a 20" circular radius aperture. Such an aperture, corresponding to $\simeq 3000 \mathrm{AU}$ at the distance of $160 \mathrm{pc}$, was chosen to include all the emission revealed by the maps at both wavelengths. The results are summarized in Table 2 .

These integrated fluxes are higher than the UKT14 fluxes measured by Sandell 1994 (by $17 \%$ and $29 \%$ at 450 and $850 \mu \mathrm{m}$, respectively, although the fluxes at $450 \mu \mathrm{m}$ agree within the error bars), something that we were already expecting due to the extended nature of the emission. Some of this emission was missed by the UKT14 observations (these observations correspond to apertures much smaller than the ones defined by ISO-LWS and IRAS). We will have the opportunity to estimate what fraction of the total emission was not detected and correct the respective UKT14 fluxes.

\section{Modelling the spectral energy distribution of IRAS 16293-2422}

Figure 4 shows the spectral energy distribution of IRAS 16293-2422 constructed with IRAS PSC (Point Source Catalog) data (open circles) and with the ISO-LWS and the SCUBA data presented in this paper (filled circles 
Table 2. SCUBA photometry of IRAS 16293-2422.

\begin{tabular}{cccc}
\hline \hline $\begin{array}{c}\text { Wavelength } \\
(\mu \mathrm{m})\end{array}$ & $\begin{array}{c}\text { Beam } \\
(\operatorname{arcsec})\end{array}$ & $\begin{array}{c}\text { Integrated flux density }\left(R=20^{\prime \prime}\right) \\
(\mathrm{Jy})\end{array}$ & $\begin{array}{c}\text { Peak flux density } \\
\left(\mathrm{Jy} \mathrm{beam}^{-1}\right)\end{array}$ \\
\hline 450 & 8.8 & $144.3 \pm 57.0$ & 89.6 \\
850 & 14.6 & $23.7 \pm 1.3$ & 16.9 \\
\hline
\end{tabular}
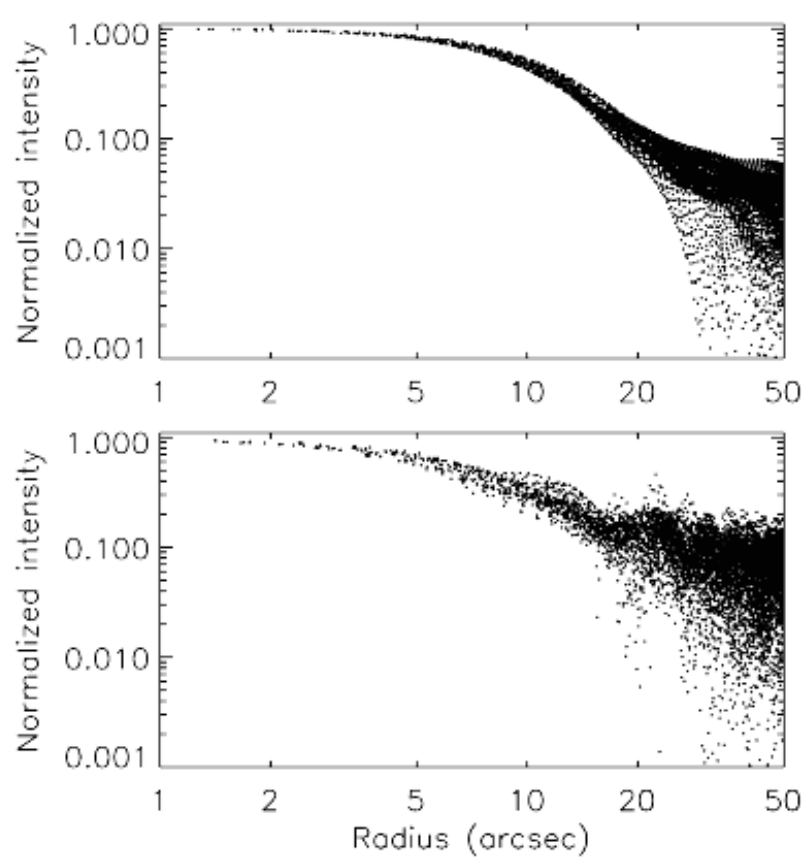

Fig. 3. Radial intensity profiles of IRAS $16293-2422$ at $850 \mu \mathrm{m}$ (upper panel) and $450 \mu \mathrm{m}$ (lower pannel). The width of the strips reflect the departure from spherical symmetry.

and open squares, respectively). We have also included the submillimetre data obtained by Sandell (1994) with UKT14 at the JCMT (filled triangles).

There is a good agreement between the IRAS-PSC data and the LWS data, especially at $100 \mu \mathrm{m}$. We can also see that the LWS is giving us access to the peak emission of IRAS 16293-2422 which is very important in defining the dust temperature. There is also an excellent agreement between the integrated fluxes obtained from the SCUBA images at 450 and $850 \mu \mathrm{m}$ (open squares) and the corresponding corrected UKT14 fluxes from the Sandell (1994) (filled triangles). The UKT14 fluxes were corrected to account for the fact that the JCMT beam did not detect all the emission, as mentioned above. The correction is explained below.

It is generally accepted that the circumstellar mass in Class 0 sources is distributed mainly in an envelope surrounding the central young stellar object (André et al. 2000; Mundy et al. 2000). Based on $\lambda=2.7 \mathrm{~mm}$ continuum interferometric measurements of IRAS 16293-2422, Looney et al. (2000) concluded that most of the flux $(\simeq 85 \%)$ is indeed in a large-scale circumbinary envelope, and that any disk flux is less than $15 \%$ of the total flux. Despite the high spatial resolution achieved,

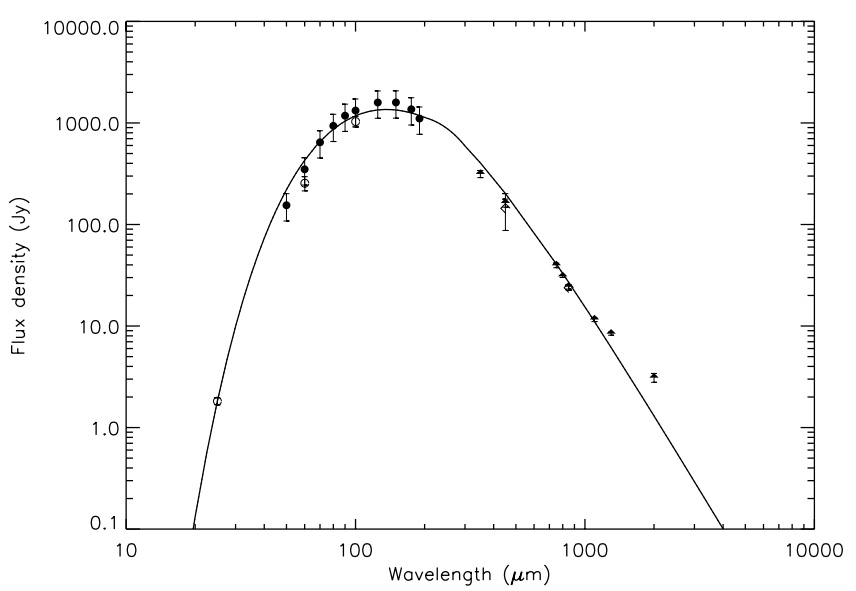

Fig. 4. The spectral energy distribution of IRAS 16293-2422 and the fit from the model obtained with $R_{\mathrm{env}}=3000 \mathrm{AU}$ and $p=2.0$ (Model 1 - see Table 3 for the other parameters). The open circles are IRAS-PSC data, the filled dots are ISO-LWS data presented in this paper, the filled triangles are data from Sandell (1994) after being corrected for the beam size effect (see text for an explanation) and the open squares are integrated fluxes in a circular aperture of 20 " from the SCUBA observations described in this paper.

the authors noted that the envelope and any possible disk contribution are difficult to disentangle.

In a more recent paper, Looney et al. (2003) could not model the inner structure of IRAS 16293-2422 due to the unresolved detection of their observations.

Considering this scenario, and although the possiblity of IRAS 16293-2422 having a disk is not ruled out, we modelled the dust emission assuming one circumstellar component, an envelope. The model consists of a spherically symmetric dust envelope with a certain radius $R_{\text {env }}$ within which the temperature $T$ and the density $\rho$ vary according to power laws, with exponents $q$ and $p$, respectively: $T(r) \propto r^{-q}$ and $\rho(r) \propto r^{-p}$. The emission from each point in the envelope is taken to be a blackbody modified by the frequency-dependent dust grain emissivity $\left(\kappa_{v} \propto v^{\beta}\right.$, where $\kappa_{v}$ is the extinction coefficient and $\beta$ is the dust opacity law coefficient). The emission is integrated along lines of sight through the envelope at a range of angular distances from the centre to determine the angular dependence of the surface brightness. The model does not assume optically thin emission but includes a radiative transfer integration along each line of sight. The total emission is computed by integrating the surface brightness over the whole envelope. Beyond the radius of the envelope, the emission is assumed to be constant and equal to the emission at the distance $R_{\text {env }}$. Figure 4 shows the fit to the data obtained with it. 
Table 3. Model parameters.

\begin{tabular}{lccccccc}
\hline \hline & $\begin{array}{c}R_{\text {env }} \\
(\mathrm{AU})\end{array}$ & $\begin{array}{c}T_{\text {env }} \\
(\mathrm{K})\end{array}$ & $\begin{array}{c}\rho_{\text {env }} \\
\left(\mathrm{g} \mathrm{cm}^{-3}\right)\end{array}$ & $q$ & $p$ & $\beta_{1}$ & $\beta_{2}$ \\
\hline Model 1 & 3000 & 19 & $6.0 \times 10^{-20}$ & 0.4 & 2.0 & 0.8 & 1.8 \\
Model 2 & 3250 & 22 & $6.0 \times 10^{-20}$ & 0.3 & 1.5 & 1.0 & 1.5 \\
\hline
\end{tabular}

As we approach the centre of the envelope, the temperature increases and we should expect not to have any dust closer to the centre than a certain distance, defined here as the inner radius of the envelope $R_{i}$. This radius is defined by the sublimation temperature of the dust $T_{\text {sub }}$. For a typical mixture of dust grains, a sublimation temperature of 1000-2000 K is normally adopted (Evans 1994). Changing this temperature between $1000 \mathrm{~K}$ and $2000 \mathrm{~K}$ does not introduce a considerable change in the final emission. This is due to the fact that such change in temperature only implies a very small change in the inner radius $R_{i}$ when compared with the size of the envelope $R_{\text {env }}$. We adopted a sublimation temperature $T_{\text {sub }}=$ $1500 \mathrm{~K}$. A sublimation temperature of this order defines an inner radius of a few tenths of an AU for typical envelope sizes and temperature distributions, making the "hole" very small when compared with the size of the envelope.

If a disk component exists, as it is suggested by highangular resolution observations (Looney et al. 2000, 2003), this disk will have to have a size much bigger than the size of the "hole" defined here by the sublimation temperature. However, even a "hole" with $10 \mathrm{AU}$ of radius will be only a very small fraction (less than 1\%) of the total size of the envelopes considered here. The influence of the inner radius of the envelope on the spectral energy distribution will be mentioned in the next section.

Based on experimental and observational works available at the time, Hildebrand (1983) considered two different values for the dust emissivity index in the far-infrared and the submillimetre regions $(\beta=1$ for $50 \mu \mathrm{m} \leq \lambda \leq 250 \mu \mathrm{m}$ and $\beta=2$ for $\lambda \geq 250 \mu \mathrm{m})$. We used the same approach but we let $\beta$ be a free parameter. Two values of $\beta$ were used in the model, $\beta_{1}$ and $\beta_{2}$, to account for different opacities in the far-infrared and submillimetre/millimetre regimes. The need for two different dust opacity indices is made clear in the next section where the influence of each parameter on the SED is studied. Although it is clear that $\beta$ should be a function of wavelength, it is not physically realistic to assume that it changes from $\simeq 1$ to $\simeq 2$ in a discontinous way. For this reason, and in order to have a smooth transition between the two regimes, we defined a region between $200 \mu \mathrm{m}$ and $300 \mu \mathrm{m}$ where $\beta$ changes from its short-wavelength value $\left(\beta_{1}\right)$ to its long-wavelength value $\left(\beta_{2}\right)$. For simplicity, the dependence of $\beta$ over the transition region was assumed to be linear. Following Hildebrand's approach, we considered spherical grains with an average radius $a=0.1 \mu \mathrm{m}$, a grain density box $\rho_{\mathrm{g}}=3 \mathrm{~g} \mathrm{~cm}^{-3}$ and an extinction efficiency at $250 \mu \mathrm{m}, Q_{0}=3.75 \times 10^{-4}$. $Q_{0}$ is believed to be good within a factor of two (Hildebrand 1983; Mezger 1990; Henning 1996).
The model shown in Fig. 4 corresponds to an envelope with a radius $R_{\mathrm{env}}=3000 \mathrm{AU}$ and a power law density distribution $p=2.0$. The remaining parameters used for the model are summarized in Table 3 under "Model 1" $\left(T_{\text {env }}\right.$ and $\rho_{\text {env }}$ are the temperature and the density of the dust at the radius $R_{\text {env }}$, respectively, $q$ is the power law temperature distribution index and $\beta_{1}$ and $\beta_{2}$ are the infrared and submillimetre/millimetre indices, respectively).

At the distance of $160 \mathrm{pc}$, the radius of the envelope $R_{\mathrm{env}}=$ 3000 AU corresponds to $\simeq 20^{\prime \prime}$ which is comparable to the UKT14 19" average beam FWH. This implies that the UKT14 observations from Sandell (1994) missed a significant fraction of the emission. From the angular dependence of the surface brightness predicted by our model we estimate that an average of $\simeq 40 \%$ of the emission from the envelope was lost by the UKT14 beam at all wavelengths. For this reason, we corrected the fluxes by multiplying them, on average, by 1.4 and plotted them together with our data to construct the SED, as shown in Fig. 4.

The fit shows that it is possible to reproduce most of the dust emission from the mid-infrared to the millimetre range of the spectrum without the need for another circumstellar component in addition to the envelope. However, the model fails to reproduce the millimetre excess detected at $1300 \mu \mathrm{m}$ and $2000 \mu \mathrm{m}$. We believe that this excess is due to the existence of the small disk not resolved by the $2.7 \mathrm{~mm}$ interferometric survey from Looney et al. (2000). Due to the difficulty in disentangling the disk and the envelope, we cannot constrain any parameters of the disk and so we have not included it in our modelling. However, we would like to point out that the inclusion of a disk in the model does not introduce a significant change in the intensity profiles. This inclusion affects significantly the slopes of the SED in the mid-infrared and in the submm regions of the spectrum, the two regions where the contribution from the envelope is not sufficient enough to mask the contribution from the disk. To model the contribution from a disk we would need high-resolution thermal infrared imaging observations.

\section{Discussion}

\subsection{The influence of the different parameters on the SED}

A brief exploration of the parameter space associated with the model used in this paper was carried out by Correia et al. (1999), and a more complete analysis was done by Correia (2000). In this paper, we show the results from running the model for different values of the radius of the envelope $\left(R_{\text {env }}\right)$, different temperature and density power law indices ( $q$ and $p$ ) and for different dust opacity indices $\left(\beta_{1}\right.$ and $\left.\beta_{2}\right)$. These results can be seen in the following figures. The parameters that gave the fit shown in Fig. 4 were used and only the parameter whose role we were studying was allowed to change.

Figure 5 shows the effect of changing $R_{\text {env }}$ from $2750 \mathrm{AU}$ to $3750 \mathrm{AU}$ at intervals of $250 \mathrm{AU}$.

As we would expect, lower values of $R_{\text {env }}$ imply lower fluxes. Increasing the radius of the envelope corresponds to 


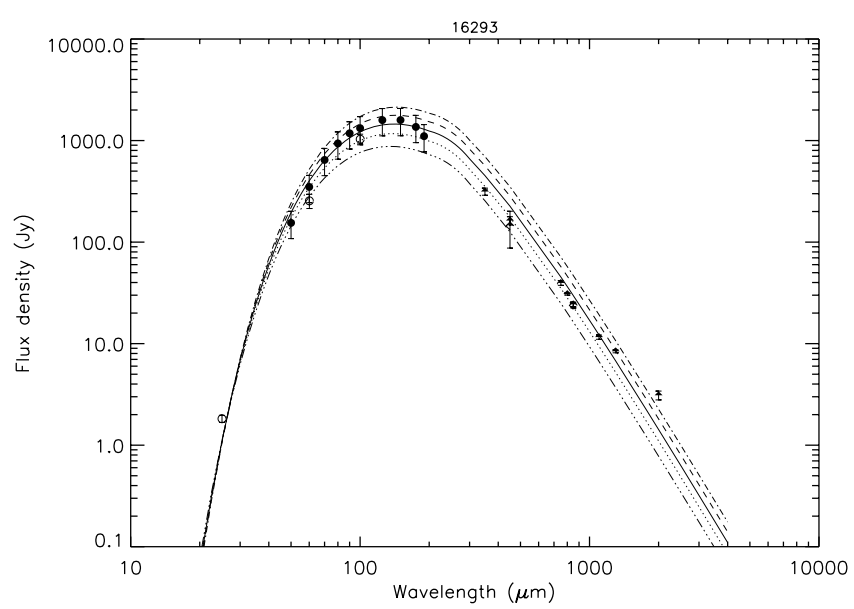

Fig. 5. Fits for different values of $R_{\mathrm{env}}$. Fits are shown for $R_{\mathrm{env}}=2500$, 2750, 3000, $3250 \mathrm{AU}$ and $3500 \mathrm{AU}$ with the solid line corresponding to the "best fit" $\left(R_{\mathrm{env}}=3000 \mathrm{AU}\right)$.

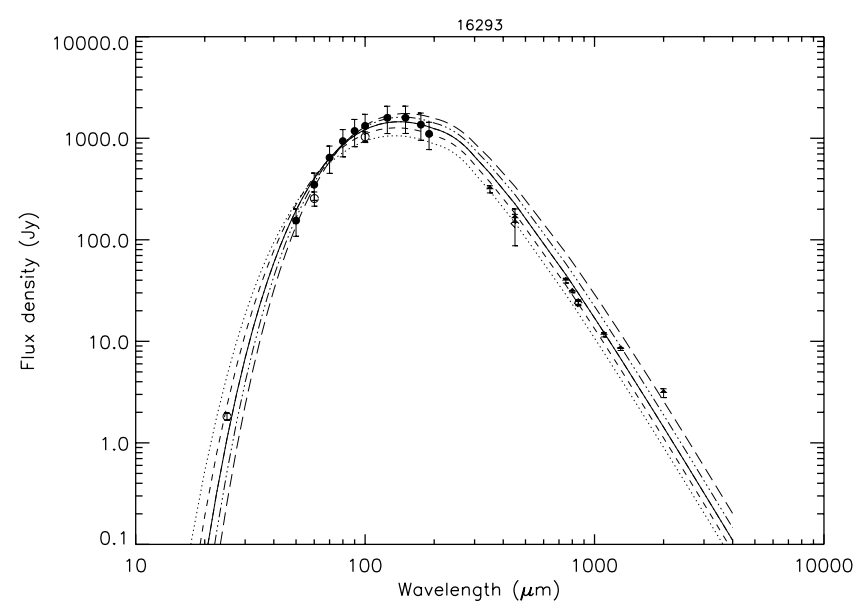

Fig. 6. Fits for different values of $p$. Fits are shown for $p=1.6$, $1.8,2.0,2.2,2.4$ with the solid line corresponding to the "best fit" $(p=2.0)$.

the introduction of more mass to the system, so the total emission increases. The submillimetre/millimetre emission goes up but the slope remains basically unchanged. The increase in the radius results essentially in a significant increase in the peak emission.

Figure 6 shows the effect of changing the density power law index from 1.6 to 2.4. Different values of $p$ give almost the same SED. The slopes are almost exactly the same, only the peak flux is slightly higher for the sharper density profiles, with the "best fit" being obtained for $p=2.0$. In fact, the effect of changing $p$ is very similar to the effect of changing $R_{\text {env }}$ (see Fig. 5). The comparison of the two figures shows us that there is a clear cross-correlation between the two parameters and that different parameter combinations could produce equally acceptable fits to the SED. We conclude that constraining these two parameters with only continuum SED data is a very difficult task, if not impossible.

Figure 7 illustrates well the effect of changing the temperature power law index.

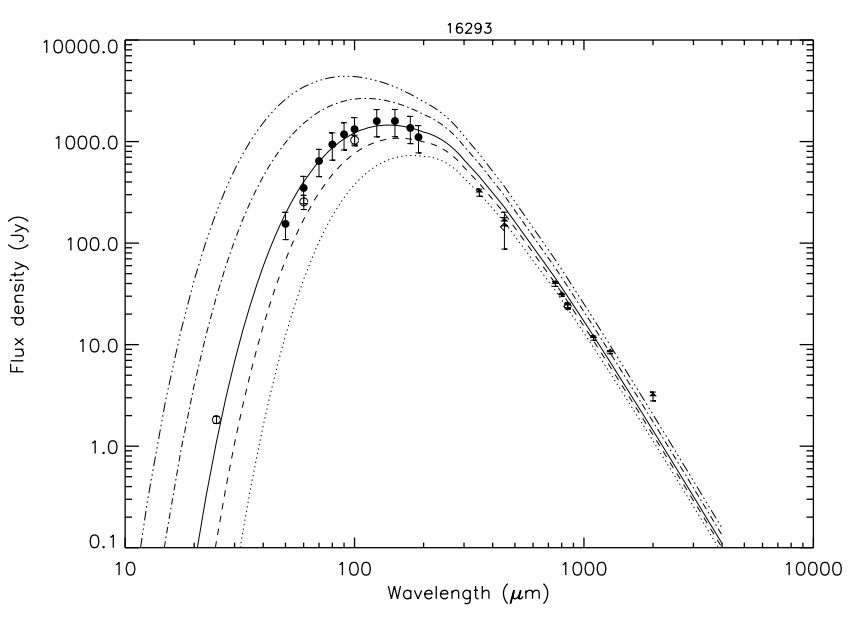

Fig. 7. Fits for different values of $q$. Fits are shown for $q=0.6,0.5$, $0.4,0.3,0.2$ with the higher values shifting the peak emission towards the short wavelengths and higher flux densities. The solid line corresponds to $q=0.4$.

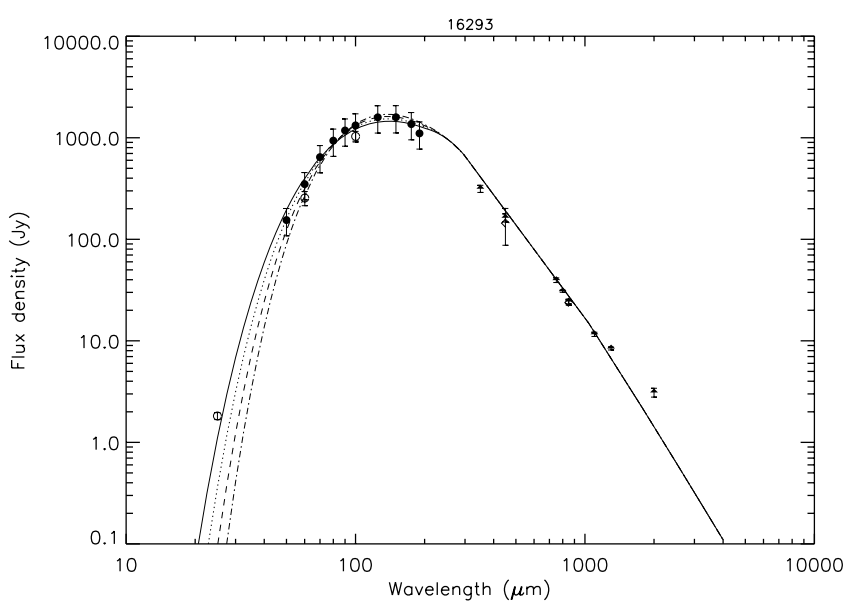

Fig. 8. Fits for different values of $\beta_{1}$. Fits are shown for $\beta_{1}=0.8$, $1.0,1.2$, and 1.4 with the solid line corresponding to the "best fit" $\left(\beta_{1}=0.8\right)$.

The slope and the fluxes in the submm/mm are not significantly affected, but very different fluxes are obtained at shorter wavelengths. Increasing $q$, which corresponds to sharper temperature profiles, means shifting the peak emission towards the short wavelengths, increasing the IR emission and broadening the SED. This figure shows how important LWS data can be. The LWS data define the peak emission of the object giving us access to the turnover of the SED which is vital in constraining the temperature power law index.

Figure 8 shows fits obtained with different values of $\beta_{1}$, ranging from 0.8 to 1.4 . Higher values of $\beta_{1}$ produce sharper SEDs, decreasing the emission below $\sim 60 \mu \mathrm{m}$ and increasing slightly the peak flux.

A comparison with Fig. 6 shows that $\beta_{1}$ is difficult to be well constrained by short wavelength far-infrared data. A similar effect is caused on the SED either by increasing $\beta_{1}$ or the density power law index $p$. This is explained by the strong dependence of the shape of the SED on the optical depth at the shortest wavelengths. 


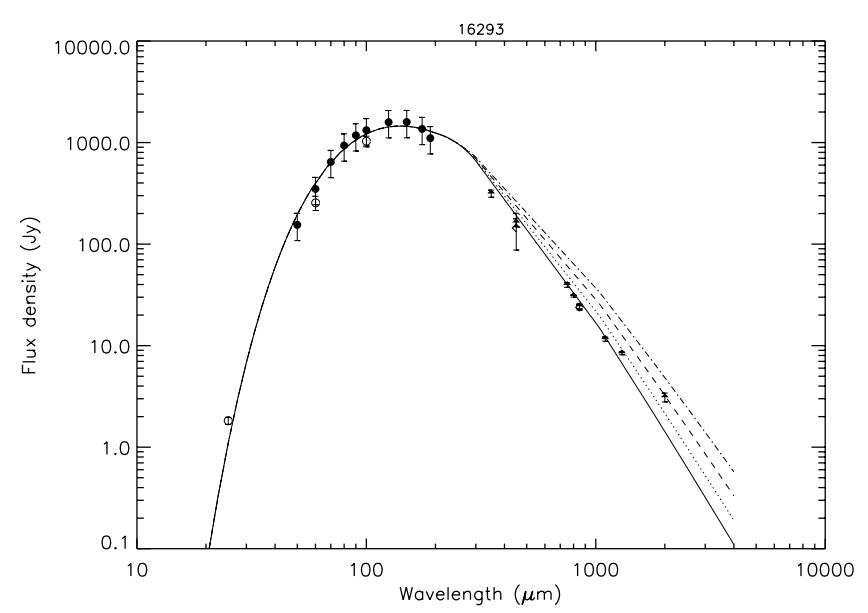

Fig. 9. Fits for different values of $\beta_{2}$. Fits are shown for $\beta_{2}=1.8$, $1.6,1.4$, and 1.2 , with the solid line corresponding to the "best fit" $\left(\beta_{2}=1.8\right)$.

A similar dependence could eventually be expected on the inner radius of the envelope. In this paper this radius was defined by the sublimation temperature. If a disk exists, then this inner radius should be larger than that assumed here. However, an examination of the influence of the inner radius on the shape of the SED has shown that only residual changes are introduced even if an inner radius of several tens of AU is considered. This is explained by the fact that even such an inner radius would be less than $1 \%$ of the total radius of the envelope. This would still define a very small inner "hole" whose emission is negligible when compared with the emission from the rest of the envelope. In this sense, the small inner radius considered here (defined by the sublimation temperature) does not exclude the existence of a disk. Due to the lack of information that could constrain such a disk and the resulting inner "hole" in the envelope (even considering the high-angular resolution data of Looney et al. 2000, 2003), we do not have data available that would allow us to change the size of the inner "hole" considered here.

The effects of changing $\beta_{2}$ are shown in Fig. 9. Decreasing the index produces higher fluxes at the submillimetre/millimetre range. The slope is clearly defined by $\beta_{2}$, meaning that $\beta_{2}$ can be very well constrained by submillimetre data. The comparison with Fig. 8 shows that two very different values of the dust opacity index have to be chosen to fit, at the same time, the infrared and the submillimetre slopes of the SED.

We conclude that the radius of the envelope and the dust density index $p$ have similar effects on the SED of the object, with larger radius and steeper density distributions implying higher emissions in the far-infrared and in the submillimetre, with the consequent higher peak emissions.

\subsection{Model degeneracy, intensity profiles and errors}

The degeneracy of the fits was studied by looking at different possible sets of parameters giving the same fit. The idea behind this approach was to analyse the ambiguities resulting from not being able to constrain each of the parameters uniquely without spatial information about the dust distribution.

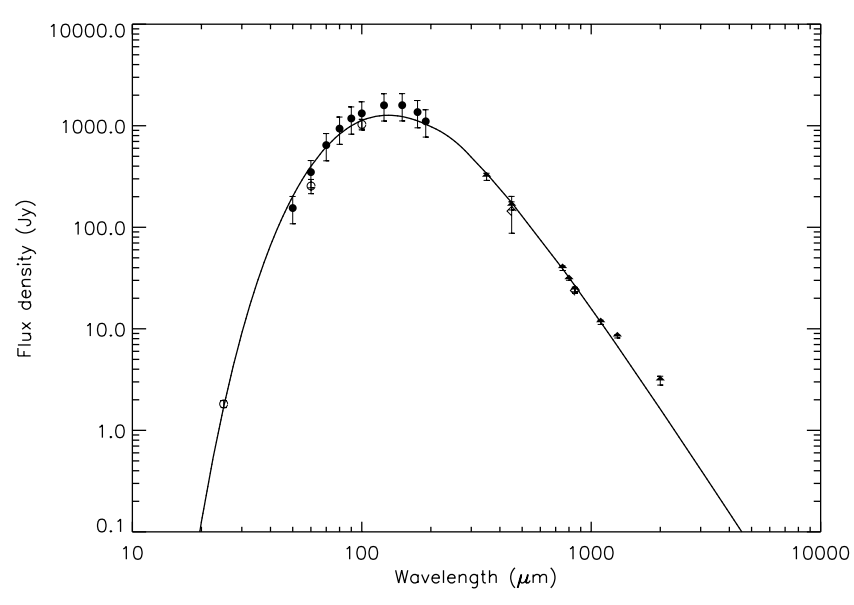

Fig. 10. The spectral energy distribution of IRAS 16293-2422 and the fit from the model obtained with $R_{\mathrm{env}}=3250 \mathrm{AU}$ and $p=1.5$ (Model 2 - see Table 1 for the other parameters). The symbols are as in Fig. 4.

To illustrate this problem, Fig. 10 shows another possible good fit to the SED obtained with $p=1.5$ and $R_{\text {env }}=$ 3250 AU (the remaining parameters are listed in Table 3 under "Model 2").

A less sharp density distribution implies lower fluxes in the far-infrared and in the submillimetre, but this effect is compensated with a larger radius, resulting in a SED very similar to the one shown in Fig. 4. This example shows that the widely adopted practice of fitting only SEDs in order to derive circumstellar parameters can be misleading and incorrect, and emphasises the need for spatial information about the object.

Figure 11 shows the intensity profiles at $850 \mu \mathrm{m}$ and $450 \mu \mathrm{m}$ predicted by Model 2 (upper and lower panels, respectively). The dotted lines shows the intensity profiles predicted by the model, the dashed lines corresponds to the Gaussian beam profile measured from the SCUBA maps of Uranus at the two wavelengths and the solid lines corresponds to the intensity profiles after a two-dimensional convolution with the respective beams. The dotted strips represents the intensity profiles of the object measured from the maps shown in Figs. 1 and 2. The figures shows that, although a good fit to the SED was obtained, the profile predicted by the model at $850 \mu \mathrm{m}$ is at the upper end of the profile derived from the SCUBA map.

Figure 12 shows the measured profiles and the intensity profiles from the model at $850 \mu \mathrm{m}$ and $450 \mu \mathrm{m}$ obtained with $p=2.0$ and $R_{\text {env }}=3000 \mathrm{AU}$ (Model 1). In this case the emission is more centrally concentrated, resulting in sharper intensity profiles. In this case, the predicted profile at $850 \mu \mathrm{m}$ is at the lower end of the measured profile. For angles greater than $20^{\prime \prime}$ this model profile is actually better than the one shown in the previous figure. The predicted profile at $450 \mu \mathrm{m}$ is a clear underestimate of the observed profile. The analysis and comparison of the two figures shows us that, just on the basis of the $850 \mu \mathrm{m}$ profiles, a value between $p=1.5$ and $p=2.0$ is the most appropriate value. The data at $450 \mu \mathrm{m}$ implies a value at the bottom end of this range. However, we call the attention to the fact that these data suffers from lower $S / N$ ratio and from 


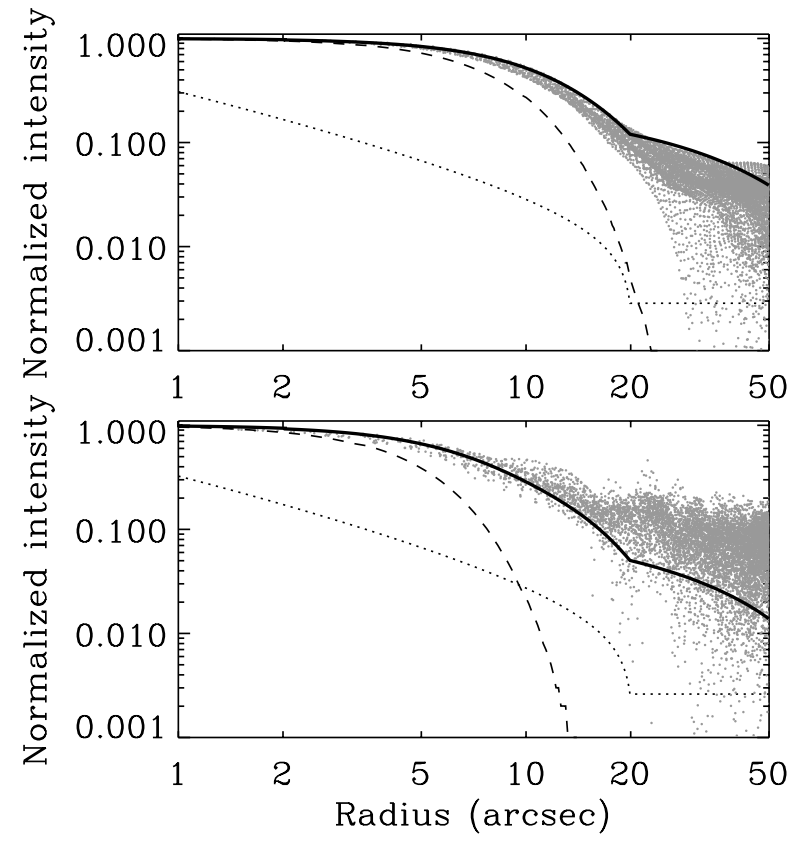

Fig. 11. Comparison of the radial intensity profiles of IRAS 16293-2422 at $850 \mu \mathrm{m}$ (upper panel) and $450 \mu \mathrm{m}$ (lower pannel) with the profiles predicted by Model $2(p=1.5$ and $\left.R_{\text {env }}=3250 \mathrm{AU}\right)$. The dotted strips correspond to the intensity profiles of the object derived from the SCUBA images. The width of the strips reflect the departure from spherical symmetry. The dotted lines show the intensity profiles predicted by the model, the dashed lines correspond to the Gaussian beam profiles measured from the SCUBA maps of Uranus and the solid lines correspond to the intensity profiles after a two-dimensional convolution with the beams.

the higher probablility that the large error beam at this wavelength can be picking up some power.

The finite chop throw is believed not to have an influence in the intensity profiles as the $120^{\prime \prime}$ used guarantees us that no significant on-source emission is included. Contamination from the $\mathrm{NH}_{3}$ core discovered by Wootten \& Loren (1987) is also discarded as mentioned in Sect. 2.2.

These results are in agreement with the idea that the envelopes of Class 0 and Class I protostars are always found to be strongly centrally condensed, with density profiles consistent with $\rho(r) \propto r^{-p}$ with $p \sim 1.5-2$ (André et al. 2000; Mundy et al. 2000). In most cases, it is difficult to distinguish between $r^{-1.5}$ and $r^{-2}$ density profiles, or to establish the radius where these gradients apply. Although the SCUBA observations presented in this paper do not allow us to eliminate completly any of the two possible density profiles, the results from out modelling tell us that the envelope of IRAS 16293-2422 is observed to be very compact and centrally peaked, with a finite radius of influence. This is in agreement with the conclusions of Motte et al. (1998): in $\rho$ Ophiuchi, the circumstellar envelopes of Class 0 and Class I protostars are found to be very compact, merging with dense cores, other envelopes or the diffuse ambient cloud at a radius $R_{\text {env }}<5000 \mathrm{AU}$.

The fact that we found two distinct values for the dust opacity index is in agreement with the idea that it is not possible

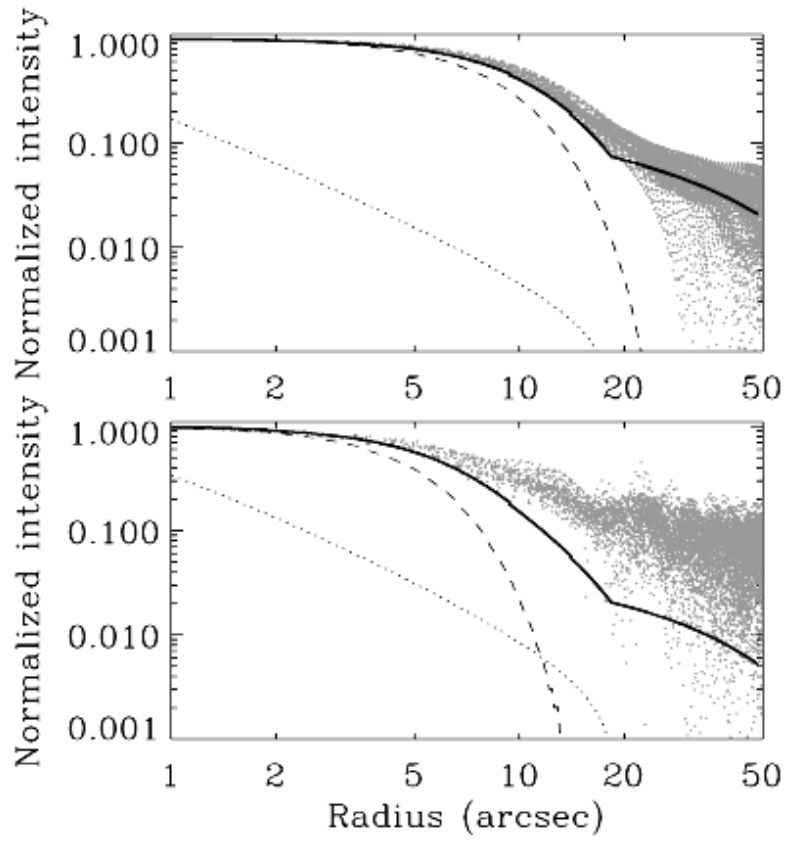

Fig. 12. Comparison of the radial intensity profiles of IRAS 16293-2422 at $850 \mu \mathrm{m}$ and $450 \mu \mathrm{m}$ (upper and lower panel, respectively) with the profiles predicted by Model 1 ( $p=2.0$ and $R_{\mathrm{env}}=3000 \mathrm{AU}$ ).

to fit all the photometric data, ranging from the infrared to the submillimetre, with only one dust opacity index valid for all the spectral range. The opacity index found for the submillimetre $\left(\beta_{2}=1.5\right)$ is too high for the infrared range of the spectrum, making the envelope optically thick. André et al. (1993) also found $\beta \simeq 1.5$ for the proto-typical Class 0 object VLA1623 and similar values have been found for other extremely young objects.

The departure from spherical symmetry, which might be explained by the presence of the two outflows associated with the source, for example, is expected to be more pronounced at shorther wavelengths. Moreover, the JCMT beam deviates from a Gaussian, especially at short wavelengths, where the main beam is superimposed on a broad error lobe (Holland 1999) making the interpretation of spatially extended maps more difficult.

It is difficult to assign any formal errors to the derived model parameters. Our phenomenological model is capable of reproducing the photometric and spatial data available to us. While a multi-parameter chi-square fit to the SED could give a mathematical solution to the problem with the respective errors associated, such an approach would be of limited use due to the large number of free parameters involved and could mislead us through providing us a mathematical solution with dubious physical meaning. For instance, our model and others like it make simplifying assumptions of spherical symmetry, power law indices for temperature and density variation, and uniform dust properties throughout the envelope. There will be departures from all these assumptions in real sources, and the results these models should therefore be interpreted with caution. 

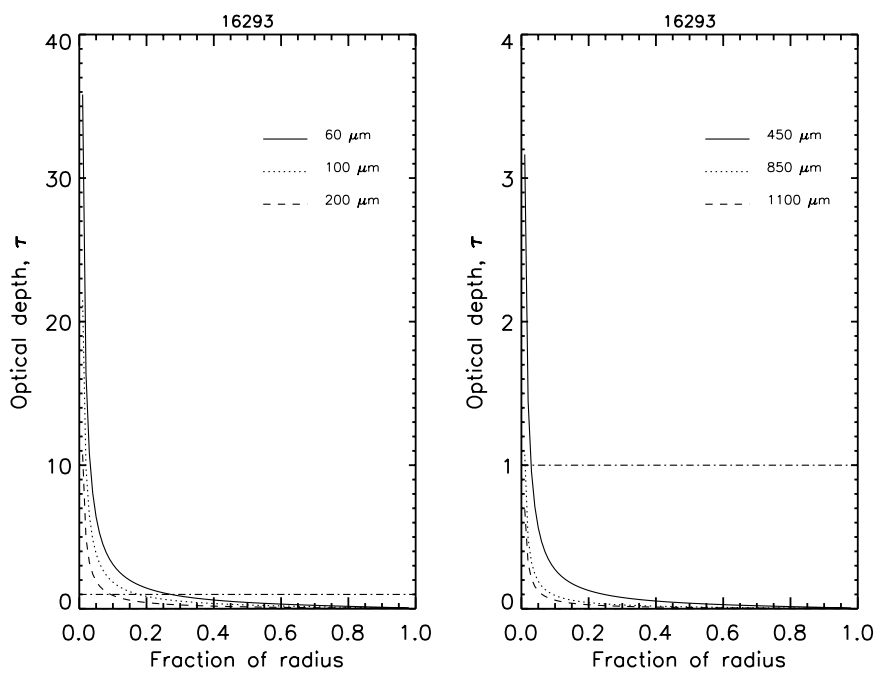

Fig. 13. IRAS 16293-2422: line of sight optical depth as a function of radius predicted by the model ( $p=2.0$ and $\left.R_{\mathrm{env}}=3000 \mathrm{AU}\right)$ at $60,100,200,450,850$ and $1100 \mu \mathrm{m}$. The horizontal line corresponds to $\tau=1$.

\subsection{Optical depth analysis}

We can compute the optical depth of the envelope for any given line of sight and at any chosen wavelength. Figure 13 shows the optical depth as a function of the distance from the centre of the envelope at six different wavelengths: $60,100,200,450,850$ and $1100 \mu \mathrm{m}$. The analysis of the figure shows that the envelope is optically thin almost over its entire volume, even at the shortest wavelengths. At $100 \mu \mathrm{m}$, for example, only the regions inside the volume defined by $\simeq 20 \%$ of the radius are optically thick. At $450 \mu \mathrm{m}$, these regions are only at a distance of $\simeq 5 \%$ of the radius. Notice, however, the extremely sharp increase in the optical depth once we approach the inner regions of the envelope. This is due to the highly centred dust distribution defined by the peaked density distribution $(p=2)$.

Figure 14 shows the optical depth as a function of wavelength for five different lines of sight along the envelope, corresponding to distances of $5,10,25,50$ and $75 \%$ of the radius of the envelope. The inflexion points that can be seen between 200 and $300 \mu \mathrm{m}$ for the lines corresponding to the inner most regions of the envelope are due to the linear change in the optical depth imposed by the change in the dust opacity index $\beta$. Figure 14 shows that the envelope, even in its innermost regions, is optically thin at wavelengths $\lambda>350 \mu \mathrm{m}$. At a distance of $25 \%$ of the radius from the centre, the dust becomes optically thick at $\lambda \simeq 70 \mu \mathrm{m}$ but at a distance of $75 \%$ the emission is optically thin over the entire range of the spectrum shown in the figure. At $100 \mu \mathrm{m}$, the inner regions of the envelope are optically thick. At $10 \mu \mathrm{m}$, even the outer regions of the envelope are optically thick. Concluding, the envelope is optically thin in the submillimetre and millimetre but it quickly becomes optically thick in the far-infrared.

Using the parameters from Model 1 and integrating the density distribution along the envelope, we estimate an envelope mass of 3.4 $M_{\odot}$ (a typical gas-to-dust ratio of 100 was assumed (Hildebrand 1983)). Integrating the emission under the model

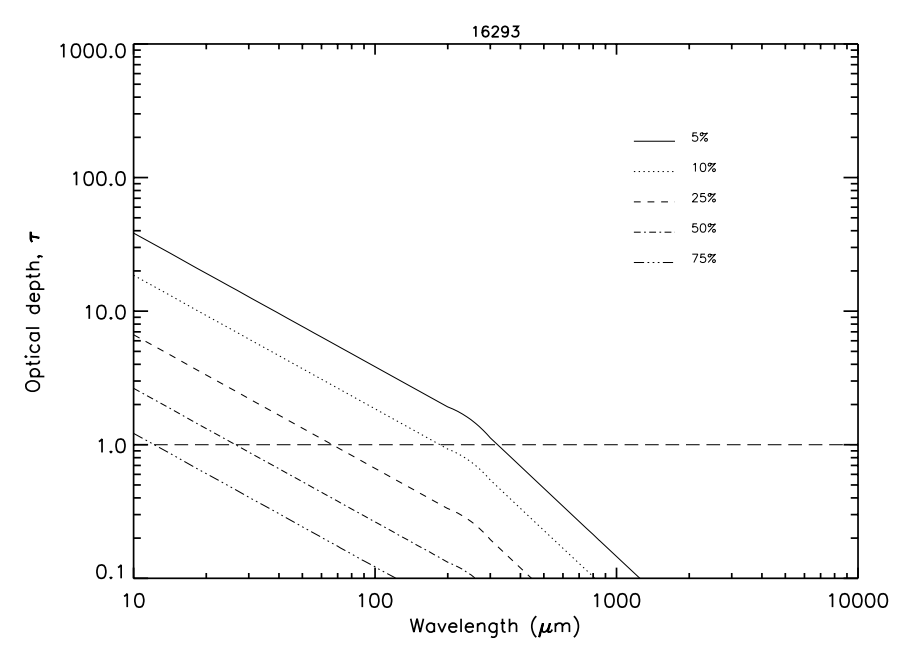

Fig. 14. IRAS 16293-2422: optical depth as a function of wavelength predicted by the model for five different lines of sight along the envelope, corresponding to distances of 5, 10, 25, 50 and $75 \%$ of the radius of the envelope. The horizontal line corresponds to $\tau=1$.

spectrum yields a bolometric luminosity of $36 L_{\odot}$ and a submillimetre luminosity of $0.7 L_{\odot}$ (defined as the luminosity radiated longward of $350 \mu \mathrm{m})$. This gives a $L_{\mathrm{submm}} / L_{\mathrm{bol}}$ ratio of $1.9 \%$, confirming the young status of the source (Class 0 sources are defined by $L_{\text {submm }} / L_{\mathrm{bol}}>0.5 \%$ (André et al. 2000)). The fact that our estimate for the bolometric luminosity is higher than the values found by Walker et al. (1990), Mezger et al. (1992) and André \& Montmerle (1994) can be explained by our better characterization of the SED, achieved by the use of LWS and SCUBA data.

\section{Conclusions}

Our main conclusions are:

1. The ISO-LWS observations of IRAS 16293-2422 presented in this paper are in good agreement with the IRAS data. These observations enabled us to define the peak emission of the object. Submillimetre imaging done with SCUBA allowed us to compute integrated fluxes at 450 and $850 \mu \mathrm{m}$. The combination of ISO and SCUBA data with IRAS data allowed us to construct a complete spectral energy distribution.

2. The SED is well fitted by a spherically symmetric envelope model. However, spatial information is essential to distinguish between different possible fits. Simultaneous fitting of the SED and the intensity profiles derived from the SCUBA observations allows us to contrain the dust envelope parameters. The SED and the $850 \mu \mathrm{m}$ intensity profile are consistent with a centrally peaked power law dust density distribution of the form $\rho(r) \propto r^{-p}$ with $p=1.5-2$, with a radius $R_{\text {env }}=3000-3250 \mathrm{AU}$, defining a very compact circumstellar envelope.

3. The following source parameters were derived: $L_{\mathrm{bol}}=$ $36 L_{\odot}, M_{\mathrm{env}}=3.4 M_{\odot}$ and $L_{\mathrm{submm}} / L_{\mathrm{bol}}=1.9 \%$, confirming that the source shows a submillimetre excess characteristic of Class 0 sources. 
Acknowledgements. The authors would like to thank Wayne Holland for his help with the SCUBA observations and data reduction, Philippe André for useful discussions and the LWS Consortium for making the ISO observations possible. We also thank the anonymous referee for careful reading and useful comments and suggestions that have improved the original manuscript. J. C. Correia gratefully acknowledges the financial support from FCT (Fundação para a Ciência e a Tecnologia - Portugal) through the grants Programa PRAXIS XXI/BD/2961/94 and SFRH/BPD/3614/2000.

\section{References}

Adams, F. C., Lada, C. J., \& Shu, F. H. 1987, ApJ, 312, 788

André, P., \& Montmerle, T. 1994, ApJ, 420, 837

André, P., Ward-Thompson, D., \& Barsony, M. 1993, ApJ, 406, 122

André, P., Ward-Thompson, D., \& Barsony, M. 2000, From Pre-Stellar Cores to Protostars: The Initial Conditions of Star Formation, Protostars and Planets IV, ed. V. Mannings, A. P. Boss, \& S. S. Russell (Tucson: University of Arizona Press)

Boss, A. P., \& Yorke, H. W. 1995, ApJ, 411, L99

Butner, H. M., Natta, A., \& Evans, N. J. 1994, ApJ, 420, 326

Ceccarelli, C., Caux, E., White, G. J., et al. 1998, A\&A, 331, 372

Clegg, P. E., Ade, P. A. R., Armand, C., et al. 1996, A\&A, 315, L38

Correia, J. C., Griffin, M., \& Saraceno, P. 1999, Protostellar Envelopes: Modelling the Dust Emission using LWS and GroundBased Submillimetre Observations, The Universe as seen by ISO, ed. P. Cox, \& M. F. Kessler (The Netherlands: ESA Publications Division), 575

Correia, J. C. 2000, Ph.D. Thesis, University of London

Evans, A. 1994, The Dusty Universe, Ellis Horwood

Fukui, Y., Sugitani, K., Takaba, H., et al. 1986, ApJ, 311, L85

Henning, Th. 1996, The Role of Dust in the Formation of Stars, ed. Siebenmorgen (Springer), 250

Hildebrand, R. H. 1983, QJRAS, 24, 267

Holland, W. S., Robson, E. I., Cunningham, C. R., et al. 1999, MNRAS, 303, 659
Jenness, T., \& Lightfoot, J. F. 1997, SURF - SCUBA Data Reduction Facility, Starlink User Manual, 216

Looney, L. W., Mundy, L. G., \& Welch, W. J. 2000, ApJ, 529, 477

Looney, L. W., Mundy, L. G., \& Welch, W. J. 2003, ApJ, accepted

Men'shchikov, A. B., \& Henning, Th. 1997, A\&A, 318, 879

Mezger, P. G. 1990, in Physics and Composition of Interstellar Matter, ed. J. Krelowski, \& B. A. Wilking (Torun: Nicolaus Copernicus University)

Mezger, P. G., Sievers, A., Zylka, R., et al. 1992, A\&A, 265, 743

Mizuno, A., Fukui, Y., Iwata, T., Nozawa, S., \& Takano, T. 1990, ApJ, 356,184

Motte, F., André, P., \& Neri, R. 1998, A\&A, 336, 150

Mundy, L. G., Wiling, B. A., \& Myers, S. T. 1986, ApJ, 311, L75

Mundy, L. G., Wootten, A., Wilking, B. A., Blake, G. A., \& Sargent, A. I. 1992, ApJ, 385, 306

Mundy, L. G., Looney, L. W., \& Welch, W. J. 2000, The Sctructure and Evolution of Envelopes and Disks in Young Stellar Systems, Protostars and Planets IV, ed. V. Mannings, A. P. Boss, \& S. S. Russell (Tucson: University of Arizona Press)

Sandell, G. 1994, MNRAS, 271, 75

Swinyard, B. M., Clegg, P. E., Ade, P. A. R., et al. 1996, A\&A, 315, L43

Thamm, E., Steinacker, J., \& Henning, Th. 1994, A\&A, 287, 493

Trams, N. 1998, IDUM - ISOLWS Data Users Manual, Issue 5.0, ISO Science Operation Team

Walker, C., Lada, C. J., Young, E. T., Margulis, M., \& Wilking, B. A. 1985, BAAS, 17, 835

Walker, C. K., Lada, C. J., Young, E. T., Maloney, P. R., \& Wilking, B. A. 1986, ApJ, 309, L47

Walker, C., Lada, C. J., Young, E. T., \& Margulis, M. 1988, ApJ, 332, 335

Walker, C. K., Adams, F. C., \& Lada, C. J. 1990, ApJ, 349, 515

Walker, C., Carlstrom, J. E., \& Bieging, J. H. 1993, ApJ, 402, 655

Whittet, D. C. B. 1974, MNRAS, 196, 469

Wootten, A. 1989, ApJ, 337, 858

Wootten, A., \& Loren, R. B. 1987, ApJ, 317, 220 